\title{
Changes in the Physicochemical Properties of Starch Syrups after Processing by Honeybees
}

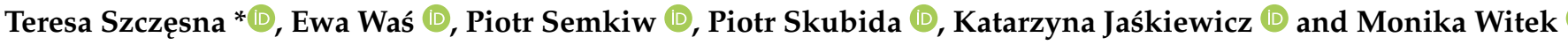 \\ The National Institute of Horticultural Research, Konstytucji 3 Maja 1/3, 96-100 Skierniewice, Poland; \\ ewa.was@inhort.pl (E.W.); piotr.semkiw@inhort.pl (P.S.); piotr.skubida@inhort.pl (P.S.); \\ katarzyna.jaskiewicz@inhort.pl (K.J.); monika.witek@inhort.pl (M.W.) \\ * Correspondence: teresa.szczesna@inhort.pl
}

Citation: Szczęsna, T.; Waś, E.;

Semkiw, P.; Skubida, P.; Jaśkiewicz, K.; Witek, M. Changes in the

Physicochemical Properties of Starch Syrups after Processing by

Honeybees. Agriculture 2021, 11, 335.

https://doi.org/10.3390/

agriculture11040335

Academic Editor: Nikola Puvača

Received: 9 March 2021

Accepted: 31 March 2021

Published: 8 April 2021

Publisher's Note: MDPI stays neutral with regard to jurisdictional claims in published maps and institutional affiliations.

Copyright: (c) 2021 by the authors. Licensee MDPI, Basel, Switzerland. This article is an open access article distributed under the terms and conditions of the Creative Commons Attribution (CC BY) license (https:// creativecommons.org/licenses/by/ $4.0 /)$.

\begin{abstract}
In recent time, it has become a common practice to use different kinds of starch syrups available on the market as a winter food for bees. However, the physicochemical composition of these syrups and their suitability for winter feeding of honeybee colonies are still insufficiently investigated. Therefore, our study analyzed the composition of three commercially available starch syrups, inverted saccharose syrup and homemade saccharose syrups, and tested winter stores processed by bees from these syrups. The following physicochemical parameters were investigated: water, sugars composition, maltodextrins (DP4-DP7) and 5-hydroxymethylfurfural (HMF) content, $\mathrm{pH}$ and free acidity, as well as electrical conductivity. We found that the sugar composition of starch syrups ensures the suitable sugar composition of winter stores processed by the bees from these syrups. Winter stores compared to fresh syrups had a high content of total fructose and glucose (50 g/100 g), and some amounts of maltose $(15 \mathrm{~g} / 100 \mathrm{~g})$, maltotriose $(4 \mathrm{~g} / 100 \mathrm{~g})$, and maltodextrins $(4 \mathrm{~g} / 100 \mathrm{~g})$. The small percentage of maltodextrins is responsible for maintaining the liquid consistency of the winter stores in honeybee combs during storage. Water content not exceeding $17.2 \%$ and adequate $\mathrm{pH}$ value (around 4.00) guarantee the stability of winter stores during storage in honeybee combs. A $\mathrm{HMF}$ content of up to $50 \mathrm{mg} / \mathrm{kg}$ was safe for bee colonies during wintering.
\end{abstract}

Keywords: honeybee colony; physicochemical composition; saccharose syrup; starch syrup; winter stores

\section{Introduction}

Appropriate nutrition plays an essential role in the development and maintenance of healthy and strong bee colonies. Pollen is a natural source of protein (especially essential amino acids), lipids (sterols), vitamins, and minerals for adequate growth and development, while nectar, honeydew, and honey are natural carbohydrate foods-essential energy sources for all activities of bees [1,2].

It is common in beekeeping practice to use not only saccharose syrups for the winter feeding of honeybees but also different kinds of carbohydrate products available on the market, such as: inverted sugar syrups, starch syrups, and high fructose corn syrups (HFCSs) [3-15]. The physicochemical composition of these products should guarantee appropriate composition and liquid consistency of the winter stores. According to Konopacka [16], water content should not be higher than $20 \%$. A syrup with a higher water content may start to ferment before bees feed on it. Dry matter should mainly consist of glucose, fructose, and sucrose. Sucrose content should be kept below 30\%. The invertase enzyme produced by bees hydrolyzes some amounts of saccharose-its concentration in winter supplies should not exceed $10 \%$. Syrups with a predominance of glucose over fructose content pose a potential risk of crystallization in honeybee combs during winter. The critical point at which a solution becomes saturated with glucose is $32 \mathrm{~g} / 100 \mathrm{~g}$ [3]. Other sugars (disaccharides, trisaccharides) should not be present, though, they might occur in low concentrations. Syrups made from starch usually contain high levels of maltose and 
maltotriose, from which glucose is formed due to the activity of bee enzymes [9]. The hydrolysis of these two sugars increases glucose content in winter stores. The content of mineral substances should not exceed $0.3 \%$ of dry matter [16]. Higher content of mineral substances and other components (e.g., oligosaccharides and polysaccharides) may be difficult to digest by the bees' digestive tract [8]. A long and cold winter with no possibility to fly may lead to cases of colony loss due to excessive load in bees' recta.

5-hydroxymethylfurfural (HMF) was noted to be toxic for honeybees [10,17]. This compound is also considered potentially carcinogenic to humans or might be metabolized by humans to potentially carcinogenic compounds [18]. HMF is a furanic compound formed in the Maillard reaction (nonenzymatic browning) as an intermediate, and in the dehydration of hexose, especially fructose, during the heat treatment of sugar-containing food products. The formation of HMF is facilitated by acidic conditions. So far, the permissible content of this compound in syrups recommended for the safe feeding of bees has not been established. Fresh honey usually contains no or very small amounts of HMF [19]. HMF concentration in honey increases during its processing by heat treatment and throughout storage [20,21]. According to Bailey [22], honey with a high HMF content increases the mortality of bees.

The effect of HMF on honeybee mortality is still being discussed and investigated. Some reports on HMF toxicity indicate that it's harmfulness depends on the time of exposure and HMF concentration [2,10,14,17,22-25]. Based on the presented results, it can be concluded that there is still insufficient knowledge of HMF toxicity and that it is impossible to establish a maximum level of HMF content safe for bees in syrups used as winter food. Capuano and Fogliano [17] suggested that HMF content not exceeding $30 \mathrm{mg} / \mathrm{kg}$ can be considered safe for bees.

Unlike starch syrups, the physicochemical composition of inverted sucrose syrups and their suitability for the winter feeding of bees is relatively well known [6,7]. Early studies on the suitability of starch syrups in beekeeping were conducted in the Netherlands [3] and in Germany [26]. Preliminary results showed that these types of syrups are useful for the winter feeding of bee colonies. However, the physicochemical composition of these products, and therefore, their suitability and safety for bees, are still insufficiently studied.

A Polish study on the winter feeding of bees with starch syrup indicated that it carries a risk to bee colony losses, especially in the case of a long and cold winter [8]. The study found a high glucose content $(38 \mathrm{~g} / 100 \mathrm{~g})$ with a relatively low content of fructose $(22 \%)$ in the winter stores of bee colonies fed with starch syrup-fructose/glucose ratio $(\mathrm{F}: \mathrm{G})=0.58$. Such high glucose content explains the crystallization of winter supplies in honeycomb cells. In addition, maltodextrins in starch syrup (3.3\%, on average) and in winter stores $(2.0 \%$, on average) were found. Maltodextrins present in winter stores during a long winter can overload bees' simple intestines. This might explain the bee colony losses that happened in Poland during the winter of 2005/2006 [8].

Other studies showed the crystallization of winter supplies produced by bees from three kinds of starch syrups with very high glucose concentrations (an average of $39 \mathrm{~g} / 100 \mathrm{~g})$ [8]. This phenomenon was explained by a high content of maltose (20\%) in fresh starch syrups which, due to the relatively early winter feeding of bees and favorable weather conditions, has been hydrolyzed to glucose causing a significant increase of this simple sugar in winter stores, accounting for the crystallization of the winter supplies in honeycomb cells.

Although the market offers a wide variety of starch syrups recommended for the winter feeding of bees, complete data on their physicochemical composition and suitability as winter food are lacking. Therefore, the aim of our study was to analyze the physicochemical composition of starch syrups and winter stores produced by bees from three commercially available starch syrups. 


\section{Materials and Methods}

\subsection{Materials}

Laboratory studies were conducted in 2012-2013 in the Bee Products Quality Testing Laboratory of the National Institute of Horticultural Research, Apiculture Division, Puławy, Poland, simultaneously to the field experiment performed by Semkiw and Skubida [12]. Three commercially available starch syrups: starch syrup I (Apifortune, manufactured in France, ICKO Apiculture, Bollène), starch syrup II (Apikel 20, manufactured in Germany, Kellman Produktions GmbH, Stendal), and starch syrup III (Apifood, manufactured in Poland, PPHU Orion-Jarosław Kasprzyk, Miedźno) were analyzed. In addition, homemade saccharose syrup which we prepared from beet sugar (sugar to water ratio 5:3) and inverted saccharose syrup (Apiinvert, manufactured in Germany, Südzucker AG, Mannheim) were also tested and used as references. Each year the studied syrups came from the same manufacturers. These syrups were used for the winter feeding of honeybee colonies of experimental apiaries of the National Institute of Horticultural Research, Apiculture Division, Puławy, Poland, over two consecutive seasons (2012 and 2013) twice: during the early feeding (from the middle of August to the end of the first decade of September) and the late feeding (from the first or the second decade of September to the end of the first decade of October). The methodology of the field experiment has been described in detail by Semkiw and Skubida [12]. Samples of the studied syrups used to feed the bee colonies and five samples of winter stores produced by the bees in each feeding period (early and late) from each of these syrups were used in the physicochemical analysis. In total, 10 samples of fresh syrups and 100 samples of winter stores produced by bees from these syrups were collected and analyzed in the laboratory.

\subsection{Methods}

The following physicochemical parameters were determined in the syrup and winter store samples: water, sugars (fructose, glucose, saccharose, turanose, maltose, trehalose, izomaltose, erlose, and maltotriose), 5-hydroksymethylfurfural (HMF), $\mathrm{pH}$, free acidity, and electrical conductivity. The above-mentioned parameters were tested according to analytical methods established by the International Honey Commission [27] and modified by Szczessna et al. [28] for honey quality assessment.

The water content was determined by a refractometric method using a thermostated Atago RX-5000 $\alpha$ digital refractometer (ATAGO CO., LTD., Tokyo, Japan). Water measurements were performed at $20^{\circ} \mathrm{C}$.

Sugars including monosaccharides (glucose and fructose), disaccharides (saccharose, turanose, maltose, trehalose, and isomaltose), and trisaccharides (rafinose, erlose, and maltotriose) were determined using a high-performance liquid chromatography technique with a refractometric detector (HPLC-RI). A Shimadzu HPLC system (SHIMADZU CO., Kyoto, Japan) consisting of an LC-10ADVP pump, CTO-10ASVP column oven, SIL-10ADVP auto injector, RID-10A refractive index detector, and PhenoSphere LC column, NH2 $80 \AA$, $250 \times 4.6 \mathrm{~mm}$ (Phenomenex Inc., Torrance, CA, USA) were used for this analysis. The HPLC system was run using computer software in the following conditions: flow rate $1.5 \mathrm{~mL} / \mathrm{min}$, mobile phase acetonitrile:water $(80: 20 \mathrm{v} / \mathrm{v})$ column and detector temperature $30^{\circ} \mathrm{C}$; and injection volume $20 \mu \mathrm{L}$. The sugars were identified through the comparison of individual sugars' retention times of the reference and the analyzed solution. The quantitative assays were done by the external standard method comparing the peak surfaces of these sugars. The results are expressed as $\mathrm{g} / 100 \mathrm{~g}$ to one decimal place.

HMF content was determined by a high-performance liquid chromatography technique with a UV detector (HPLC-RI). A Knauer HPLC system (KNAUER GmbH, Berlin, Germany) consisting of HPLC K-501 and K-1001 pumps, a degasser, dynamic mixing chamber, 3800 autosampler and UV K-2501 detector, and Eurospher $100 \AA$, 5 m C18 vertex plus column (BGB Analytik Vertrieb GmbH, Lörrach, Germany) were used for this analysis. An external standard method for the quantitative analysis of HMF was applied. 
Free acidity was determined by the potentiometric titration method and $\mathrm{pH}$ was determined by the potentiometric method using a DL50 Titrator equipped with a Rondolino autosampler (Mettler-Toledo GmbH, Greifensee, Switzerland).

Electrical conductivity was determined by the conductometric method using a WTW inoLAB Cond 700 conductometer (WTW, Weilheim, Germany). The results for electrical conductivity were calculated using a temperature correction factor of $2.6 \% /{ }^{\circ} \mathrm{C}$.

The analyses of maltodextrins were performed with high-performance liquid chromatography using a refractometric detector (HPLC-RI) following the method elaborated by Rybak-Chmielewska et al. [8]. Maltodextrins composed of 4 to 7 glucose molecules (maltotetraose (DP4), maltopentaose (DP5), maltohexaose (DP6), and maltoheptaose (DP7)) were determined. Chromatographic separation was performed using the Shimadzu HPLC system (the same as for sugar analysis) and the Luna column, $5 \mu \mathrm{m} \mathrm{NH} \mathrm{NH}_{2} 100 \AA, 250 \times 4.60 \mathrm{~mm}$ (Phenomenex Inc., Torrance, CA, USA). The mobile phase acetonitrile:water $(65: 35 v / v)$, flow rate $3 \mathrm{~mL} / \mathrm{min}$, and temperature of column and detector $40{ }^{\circ} \mathrm{C}$ were applied for maltodextrins separation. The quantitative analyses of maltodextrins (DP4-DP7) were performed by the external standard method.

\subsection{Statistical Analysis}

Statistical calculations were done using one-way ANOVA. The mean values of the physicochemical parameters of winter stores produced from different syrups and at different feeding periods in the two beekeeping season were compared using the Duncan test. The data for the water content were transformed using the $\operatorname{ArcSin}(x)$ function. Value $p<0.05$ indicated statistically significant differences. All the statistical calculations were done using the Statistica ver.10 software [29].

\section{Results}

\subsection{Physicochemical Composition of Fresh Syrups}

Some of the physicochemical parameters of the starch syrups (Apifortune, Apikel 20, and Apifood) and inverted saccharose syrup (Apiinvert) recommended as winter food for bee colonies used in our study (Tables 1-4) slightly differed from the values declared by the manufacturers on the product labels, as described by Semkiw and Skubida [12]. The studied syrups were characterized by a slightly lower water content. These differences were also found in the composition of sugars, maltose, and maltotriose in the starch syrups, and in saccharose for inverted sucrose sugars. The starch maltodextrins (DP4-DP7) were identified in starch syrups in amounts of $4.0 \mathrm{~g} / 100 \mathrm{~g}$, on average. Manufacturers had not declared maltodextrin amounts in starch products recommended as winter food for bees.

\subsection{Physicochemical Composition of Winter Stores Produced by Bees from Starch Syrups}

Physicochemical parameters of winter stores were significantly different in comparisons to the composition of starch syrups from which these stores were produced by bees (Tables 1-3).

In comparison to the fresh product, the winter food stores processed in the 2012 season with Apifortune syrup had a water content $31 \%$ lower in stores originating from the early feeding and $25 \%$ lower in stores from the late feeding, on average (Table 1). In the season of 2013, a similar decrease in the water content was also observed. The total of fructose and glucose content in 2012 increased by 16\% (early feeding) and by 19\% (late feeding), on average, and by $22 \%$ in 2013 (early and late feeding), on average. The ratio of fructose to glucose content (F:G) ranged on average from 0.75 (early feeding) to 0.77 (late feeding) in 2012 and from 0.73 (early feeding) to 0.78 (late feeding) in 2013. The mean F:G ratio of winter stores (0.76) was at the same level as in the fresh syrup (0.77). The maltose content decreased on average by $23 \%$ (early feeding) and by $19 \%$ (late feeding) in 2012, and by $25 \%$ and $27 \%$, respectively, in 2013. The maltotriose content decreased on average by $60 \%$ (early feeding) and by $15 \%$ (late feeding) in 2012, and by $18 \%$ and $8 \%$, respectively, in 2013. The higher decrease in maltotriose content was noticed after the early feeding in 
both years of the study. Maltodextrins were also detected; their content averaged around $4.5 \mathrm{~g} / 100 \mathrm{~g}$ (in stores from the late feeding) and around $4.9 \mathrm{~g} / 100 \mathrm{~g}$ (in stores from the early feeding) in both experimental seasons. The average HMF content was lower by $80 \%$ (early feeding) and by $73 \%$ (late feeding) in the 2021 season. A slightly higher decrease in the HMF concentration was found in 2013 , by 86 and $80 \%$, on average, in the stores produced from the early and late feeding, respectively. The mean value of electrical conductivity was 5-7 times higher than that of fresh syrup $(0.02 \mathrm{mS} / \mathrm{cm})$. Free acidity increased from $4.9 \mathrm{mval} / \mathrm{kg}$ (in the fresh syrup) to $11.7 \mathrm{mval} / \mathrm{kg}$ (early feeding) and to $14.3 \mathrm{mval} / \mathrm{kg}$ (late feeding), on average, in the winter stores from 2012; and from $1.2 \mathrm{mval} / \mathrm{kg}$ (in the fresh syrup) to $8.5 \mathrm{mval} / \mathrm{kg}$ and to $6.3 \mathrm{mval} / \mathrm{kg}$, on average, respectively, in the winter stores from the early and late feeding in 2013. The $\mathrm{pH}$ value decreased from 4.11 (in the fresh syrup) to 3.95, on average, in the winter stores from 2012 and from 4.53 (in the fresh syrup) to 3.90 (early feeding) and to 4.16 (late feeding), on average, in the winter stores from 2013.

Table 1. Physicochemical composition of winter stores produced by bees fed with starch syrup I (Apifortune).

\begin{tabular}{|c|c|c|c|c|c|c|c|c|c|c|}
\hline \multirow{3}{*}{ Physicochemical Parameter } & \multicolumn{5}{|c|}{ Feeding Period in 2012} & \multicolumn{5}{|c|}{ Feeding Period in 2013} \\
\hline & \multirow{2}{*}{$\begin{array}{l}\text { Fresh } \\
\text { Syrup }\end{array}$} & \multicolumn{2}{|c|}{ Early $(n=5)$} & \multicolumn{2}{|c|}{ Late $(n=5)$} & \multirow{2}{*}{$\begin{array}{l}\text { Fresh } \\
\text { Syrup }\end{array}$} & \multicolumn{2}{|c|}{ Early $(n=5)$} & \multicolumn{2}{|c|}{ Late $(n=5)$} \\
\hline & & From-to & Mean & From-to & Mean & & From-to & Mean & From-to & Mean \\
\hline Water (\%) & 22.9 & $15.4-16.7$ & $15.8 \mathrm{a} * *$ & $15.6-18.6$ & $17.2 b^{* *}$ & 22.7 & $14.4-16.9$ & $16.1 \mathrm{ab} * *$ & $15.2-16.0$ & $15.6 a^{* *}$ \\
\hline Electrical conductivity $(\mathrm{mS} / \mathrm{cm})$ & 0.02 & $0.08-0.13$ & $0.10 \mathrm{a}$ & $0.10-0.22$ & $0.13 \mathrm{a}$ & 0.01 & $0.10-0.20$ & $0.14 \mathrm{a}$ & $0.09-0.16$ & $0.13 \mathrm{a}$ \\
\hline Free acids (mval/kg) & 4.9 & $10.4-14.2$ & $11.7 \mathrm{bc}$ & $10.8-21.5$ & $14.3 \mathrm{c}$ & 1.2 & $6.4-10.7$ & $8.5 \mathrm{ab}$ & $5.5-6.7$ & $6.3 \mathrm{a}$ \\
\hline $\mathrm{pH}$ & 4.11 & $3.87-4.05$ & $3.94 a$ & $3.94-3.98$ & $3.96 \mathrm{a}$ & 4.53 & $3.75-4.02$ & $3.90 \mathrm{a}$ & $4.05-4.29$ & $4.16 \mathrm{~b}$ \\
\hline Fructose (g/100 g) & 18.2 & $20.2-23.4$ & $21.5 \mathrm{a}$ & $20.7-23.6$ & $21.6 \mathrm{a}$ & 18.2 & $20.5-22.3$ & $21.6 \mathrm{a}$ & $21.0-23.9$ & $22.4 \mathrm{a}$ \\
\hline Glucose (g/100 g) & 23.5 & $28.1-29.1$ & $28.7 \mathrm{ab}$ & $26.9-29.2$ & $28.1 \mathrm{a}$ & 23.6 & $28.6-30.8$ & $29.5 b$ & $28.1-29.4$ & $28.6 \mathrm{ab}$ \\
\hline Sucrose (g/100 g) & n.d. ${ }^{*}$ & n.d. *-1.8 & - & n.d. * & - & n.d. ${ }^{*}$ & n.d. * & - & n.d. $*-2.7$ & - \\
\hline Maltose (g/100 g) & 16.5 & $10.9-14.3$ & $12.7 \mathrm{ab}$ & $12.2-14.0$ & $13.3 b$ & 15.8 & $11.2-12.7$ & $11.9 \mathrm{ab}$ & $10.0-13.4$ & $11.6 \mathrm{a}$ \\
\hline Erlose (g/100 g) & n.d. * & n.d. $*-0.6$ & - & n.d. * & - & n.d. * & n.d. * & - & n.d. *-1.6 & - \\
\hline Maltotriose (g/100 g) & 5.5 & $1.7-3.1$ & $2.2 \mathrm{a}$ & $4.1-5.1$ & $4.7 \mathrm{c}$ & $4.9 \mathrm{c}$ & $3.6-4.3$ & $4.0 \mathrm{~b}$ & $3.9-5.2$ & $4.5 \mathrm{bc}$ \\
\hline $\begin{array}{l}\text { Total of fructose and glucose } \\
\qquad(\mathrm{g} / 100 \mathrm{~g})\end{array}$ & 41.7 & $41.5-51.1$ & $48.2 \mathrm{a}$ & $48.1-52.8$ & $49.7 \mathrm{a}$ & 41.8 & $49.3-52.3$ & $51.2 \mathrm{a}$ & $49.5-52.6$ & $50.9 a$ \\
\hline Fructose to glucose ratio (F:G) & 0.77 & $0.69-0.83$ & $0.75 \mathrm{a}$ & $0.74-0.81$ & $0.77 \mathrm{a}$ & 0.77 & $0.67-0.78$ & $0.73 a$ & $0.71-0.83$ & $0.78 \mathrm{a}$ \\
\hline $\begin{array}{l}\text { Total of determined sugars } \\
\qquad(\mathrm{g} / 100 \mathrm{~g})\end{array}$ & 63.7 & $63.1-68.2$ & $65.6 \mathrm{a}$ & $66.7-69.1$ & $67.6 a$ & 63.0 & $66.3-68.2$ & $67.4 \mathrm{ab}$ & $68.4-71.8$ & $69.6 c$ \\
\hline $\begin{array}{l}\text { Maltodextrins (DP4-DP7) } \\
(\mathrm{g} / 100 \mathrm{~g})\end{array}$ & 4.9 & $4.4-5.3$ & $4.9 \mathrm{a}$ & $3.9-4.9$ & $4.5 \mathrm{a}$ & 4.8 & $4.5-5.3$ & $4.8 \mathrm{a}$ & $3.7-5.0$ & $4.4 \mathrm{a}$ \\
\hline $\begin{array}{l}\text { 5-hydroxymethylfurfural (HMF) } \\
\qquad(\mathrm{mg} / \mathrm{kg})\end{array}$ & 43.3 & $5.2-11.5$ & $8.7 \mathrm{~b}$ & $10.1-15.1$ & $11.7 \mathrm{c}$ & 39.5 & $2.4-7.1$ & $5.6 a$ & $6.0-9.5$ & 7.9ab \\
\hline
\end{tabular}

* not detected at limit of detection $0.2 \mathrm{~g} / 100 \mathrm{~g} ;{ }^{* *}$ data for water content were transformed with $\operatorname{ArcSin}(\mathrm{x})$ function; $n$ : number of samples in a group of winter stores produced by bees in each feeding period (early and late) from each type of syrup; one-way analysis of variance (ANOVA) with Duncan's test were used. Different letters $(\mathrm{a}, \mathrm{b}, \mathrm{c})$ at mean values indicate statistically significant differences at $p \leq 0.05$.

Table 2. Physicochemical composition of winter stores produced by bees fed with starch syrup II (Apikel 20).

\begin{tabular}{|c|c|c|c|c|c|c|c|c|c|c|}
\hline \multirow{3}{*}{ Physicochemical Parameter } & \multicolumn{5}{|c|}{ Feeding Period in 2012} & \multicolumn{5}{|c|}{ Feeding Period in 2013} \\
\hline & \multirow{2}{*}{$\begin{array}{l}\text { Fresh } \\
\text { Syrup }\end{array}$} & \multicolumn{2}{|c|}{ Early $(n=5)$} & \multicolumn{2}{|c|}{ Late $(n=5)$} & \multirow{2}{*}{$\begin{array}{l}\text { Fresh } \\
\text { Syrup }\end{array}$} & \multicolumn{2}{|c|}{ Early $(n=5)$} & \multicolumn{2}{|c|}{ Late $(n=5)$} \\
\hline & & From-to & Mean & From-to & Mean & & From-to & Mean & From-to & Mean \\
\hline Water $(\%)$ & 23.7 & $15.7-17.1$ & $16.7 \mathrm{~b} * *$ & $16.4-18.4$ & $16.9 \mathrm{~b} * *$ & 23.6 & $15.0-17.0$ & $15.6 a^{* *}$ & $16.1-17.7$ & $16.9 \mathrm{~b} * *$ \\
\hline Electrical conductivity $(\mathrm{mS} / \mathrm{cm})$ & 0.01 & $0.07-0.12$ & $0.09 \mathrm{a}$ & $0.09-0.29$ & $0.16 \mathrm{ab}$ & 0.02 & $0.01-0.13$ & $0.09 \mathrm{a}$ & $0.10-0.22$ & $0.17 \mathrm{~b}$ \\
\hline Free acids (mval $/ \mathrm{kg}$ ) & 5.5 & $9.8-12.4$ & $10.9 a$ & $11.2-28.4$ & $16.4 \mathrm{~b}$ & 1.2 & $5.3-7.9$ & $6.8 \mathrm{a}$ & $6.6-8.5$ & $7.8 \mathrm{a}$ \\
\hline $\mathrm{pH}$ & 4.35 & $3.91-3.99$ & $3.97 \mathrm{~b}$ & $3.90-4.06$ & $3.93 b$ & 4.89 & $3.73-3.86$ & $3.80 \mathrm{a}$ & $3.98-4.39$ & $4.20 \mathrm{c}$ \\
\hline Fructose $(\mathrm{g} / 100 \mathrm{~g})$ & 11.7 & $12.7-14.6$ & $13.7 \mathrm{a}$ & $14.7-24.6$ & $18.3 b c$ & 13.5 & $15.6-16.6$ & $16.0 \mathrm{ab}$ & $17.1-22.1$ & $20.0 \mathrm{c}$ \\
\hline Glucose (g/100 g) & 16.3 & $25.3-27.4$ & $26.4 a$ & $22.9-30.8$ & $27.0 \mathrm{a}$ & 21.6 & $27.0-30.9$ & $29.1 \mathrm{a}$ & $25.5-30.0$ & $27.7 \mathrm{a}$ \\
\hline Sucrose (g/100 g) & n.d. ${ }^{*}$ & n.d. * & - & n.d. * & - & n.d. ${ }^{*}$ & n.d. * & - & n.d. $*-7.8$ & - \\
\hline Maltose (g/100 g) & 34.6 & $22.2-24.3$ & $23.1 b$ & $14.7-29.1$ & $21.1 b$ & 21.7 & $16.8-21.1$ & $18.4 \mathrm{ab}$ & $11.0-16.0$ & $13.9 \mathrm{a}$ \\
\hline Erlose $(\mathrm{g} / 100 \mathrm{~g})$ & n.d. * & n.d. * & - & n.d. * & - & n.d. * & n.d.. * & - & $1.0-3.3$ & 1.7 \\
\hline Maltotriose $(\mathrm{g} / 100 \mathrm{~g})$ & 8.8 & $2.9-3.3$ & $3.2 \mathrm{a}$ & $2.9-7.3$ & $6.0 \mathrm{~b}$ & 7.6 & $6.4-7.4$ & $6.7 \mathrm{~b}$ & $4.2-6.9$ & $5.7 \mathrm{~b}$ \\
\hline $\begin{array}{l}\text { Total of fructose and glucose } \\
\qquad(\mathrm{g} / 100 \mathrm{~g})\end{array}$ & 28.0 & $38.6-41.4$ & $40.2 \mathrm{a}$ & $38.4-54.6$ & $45.3 \mathrm{ab}$ & 35.1 & $42.6-46.6$ & $45.1 \mathrm{ab}$ & $47.1-48.0$ & $47.7 \mathrm{~b}$ \\
\hline Fructose to glucose ratio $(\mathrm{F}: \mathrm{G})$ & 0.72 & $0.47-0.55$ & $0.52 \mathrm{a}$ & $0.56-0.82$ & $0.67 \mathrm{~b}$ & 0.63 & $0.51-0.58$ & $0.55 a$ & $0.57-0.87$ & $0.72 b$ \\
\hline $\begin{array}{l}\text { Total of determined sugars } \\
\qquad(\mathrm{g} / 100 \mathrm{~g})\end{array}$ & 71.4 & $64.3-69.0$ & $66.4 \mathrm{a}$ & $75.7-80.2$ & $77.9 \mathrm{c}$ & 68.3 & $69.1-71.1$ & $70.5 b$ & $77.0-74.4$ & $72.3 b$ \\
\hline $\begin{array}{c}\text { Maltodextrins (DP4-DP7) } \\
(\mathrm{g} / 100 \mathrm{~g})\end{array}$ & 3.6 & $3.7-3.8$ & $3.7 \mathrm{ab}$ & $2.2-3.6$ & $3.3 a$ & 3.9 & $4.1-4.4$ & $4.2 \mathrm{~b}$ & $2.4-3.9$ & $3.2 \mathrm{a}$ \\
\hline $\begin{array}{l}\text { 5-hydroxymethylfurfural (HMF) } \\
\text { (mg/kg) }\end{array}$ & 14.6 & $2.0-4.2$ & $3.2 \mathrm{a}$ & $3.2-5.7$ & $4.3 \mathrm{ab}$ & 30.1 & $4.8-7.6$ & $5.7 \mathrm{~b}$ & $6.6-9.9$ & $8.3 c$ \\
\hline
\end{tabular}

${ }^{*}$ not detected at limit of detection $0.2 \mathrm{~g} / 100 \mathrm{~g} ;{ }^{* *}$ data for water content were transformed with ArcSin(x) function; $n$ : number of samples in a group of winter stores produced by bees in each feeding period (early and late) from each type of syrup; one-way analysis of variance (ANOVA) with Duncan's test were used. Different letters $(\mathrm{a}, \mathrm{b}, \mathrm{c})$ at mean values indicate statistically significant differences at $p \leq 0.05$. 
Table 3. Physicochemical composition of winter stores produced by bees fed with starch syrup III (Apifood).

\begin{tabular}{|c|c|c|c|c|c|c|c|c|c|c|}
\hline \multirow{3}{*}{ Physicochemical Parameter } & \multicolumn{5}{|c|}{ Feeding Period in 2012} & \multicolumn{5}{|c|}{ Feeding Period in 2013} \\
\hline & \multirow{2}{*}{$\begin{array}{l}\text { Fresh } \\
\text { Syrup }\end{array}$} & \multicolumn{2}{|c|}{ Early $(n=5)$} & \multicolumn{2}{|c|}{ Late $(n=5)$} & \multirow{2}{*}{$\begin{array}{l}\text { Fresh } \\
\text { Syrup }\end{array}$} & \multicolumn{2}{|c|}{ Early $(n=5)$} & \multicolumn{2}{|c|}{ Late $(n=5)$} \\
\hline & & From-to & Mean & From-to & Mean & & From-to & Mean & From-to & Mean \\
\hline Water $(\%)$ & 19.8 & $16.6-17.4$ & $17.1 b^{* *}$ & $15.6-16.9$ & $16.2 \mathrm{a}^{* *}$ & 20.9 & $15.8-17.5$ & $16.6 \mathrm{ab} * *$ & $15.6-17.5$ & $16.5 \mathrm{ab} * *$ \\
\hline Electrical conductivity (mS/cm) & 0.01 & $0.07-0.09$ & $0.08 \mathrm{a}$ & $0.10-0.15$ & $0.12 \mathrm{ab}$ & 0.01 & $0.08-0.30$ & $0.15 \mathrm{ab}$ & $0.06-0.28$ & $0.17 \mathrm{~b}$ \\
\hline Free acids (mval $/ \mathrm{kg})$ & 4.0 & $8.7-10.6$ & $9.8 \mathrm{ab}$ & $9.5-14.7$ & $12.0 \mathrm{~b}$ & 1.3 & $5.5-18.8$ & $9.9 \mathrm{ab}$ & $4.2-9.4$ & $7.2 \mathrm{a}$ \\
\hline $\mathrm{pH}$ & 4.21 & $3.90-4.06$ & $3.98 \mathrm{a}$ & $3.85-4.07$ & $3.98 \mathrm{a}$ & 4.46 & $3.80-3.95$ & $3.88 \mathrm{a}$ & $4.02-4.45$ & $4.25 b$ \\
\hline Fructose $(\mathrm{g} / 100 \mathrm{~g})$ & 20.1 & 20.9-21.6 & $21.3 \mathrm{a}$ & $17.7-23.2$ & $20.9 \mathrm{a}$ & 21.0 & $21.9-28.1$ & $24.2 \mathrm{~b}$ & $22.5-26.3$ & $24.7 \mathrm{~b}$ \\
\hline Glucose (g/100 g) & 25.2 & $28.8-31.9$ & 30.3ab & $24.0-30.5$ & $28.2 \mathrm{a}$ & 27.3 & $28.6-32.3$ & $30.9 b$ & $28.0-30.3$ & $29.6 \mathrm{ab}$ \\
\hline Sucrose (g/100 g) & n.d. * & n.d. *-1.3 & - & n.d. * & - & n.d. * & n.d * & - & n.d. ${ }^{*}-3.2$ & - \\
\hline Maltose (g/100 g) & 23.1 & $13.5-6.3$ & $14.8 \mathrm{ab}$ & $15.2-25.9$ & $18.5 b$ & 15.9 & $8.1-15.0$ & $12.0 \mathrm{a}$ & $9.7-14.7$ & $11.5 \mathrm{a}$ \\
\hline Erlose $(\mathrm{g} / 100 \mathrm{~g})$ & 1.6 & n.d. * & - & n.d. * & - & n.d. * & n.d. * & - & n.d. ${ }^{*}-1.4$ & - \\
\hline Maltotriose (g/100 g) & 7.2 & $2.0-2.5$ & $2.3 \mathrm{a}$ & $3.7-6.3$ & $5.1 \mathrm{bc}$ & 6.6 & $4.6-6.6$ & $5.8 \mathrm{c}$ & $3.5-6.0$ & $4.5 b$ \\
\hline $\begin{array}{l}\text { Total of fructose and glucose } \\
\qquad(\mathrm{g} / 100 \mathrm{~g})\end{array}$ & 45.3 & $50.4-53.1$ & $51.6 \mathrm{ab}$ & $41.7-53.7$ & $49.2 \mathrm{a}$ & 48.3 & $50.5-60.4$ & $55.1 \mathrm{~b}$ & $52.0-56.3$ & $54.3 b$ \\
\hline Fructose to glucose ratio $(\mathrm{F}: \mathrm{G})$ & 0.80 & $0.66-0.75$ & 0.70a & $0.70-0.76$ & $0.74 \mathrm{ab}$ & 0.77 & $0.73-0.87$ & $0.78 b c$ & $0.75-0.88$ & $0.84 \mathrm{c}$ \\
\hline $\begin{array}{l}\text { Total of determined sugars } \\
\qquad(\mathrm{g} / 100 \mathrm{~g})\end{array}$ & 77.2 & $67.7-69.7$ & 69.1a & $71.3-74.9$ & $73.1 b c$ & 73.4 & $72.1-73.6$ & $72.9 b$ & $73.3-75.6$ & $74.4 \mathrm{c}$ \\
\hline $\begin{array}{l}\text { Maltodextrins (DP4-DP7) } \\
\text { (g/100 g) }\end{array}$ & 3.4 & $3.1-3.6$ & $3.4 \mathrm{~b}$ & $3.2-3.7$ & $3.5 b$ & 3.2 & $2.3-3.3$ & $2.9 \mathrm{a}$ & $2.4-3.3$ & $2.7 \mathrm{a}$ \\
\hline $\begin{array}{l}\text { 5-hydroxymethylfurfural (HMF) } \\
\text { (mg/kg) }\end{array}$ & 27.2 & $2.8-4.8$ & $3.6 a$ & $3.2-8.1$ & $5.7 \mathrm{a}$ & 51.2 & $6.1-11.8$ & $10.2 b$ & $5.1-19.8$ & $14.3 \mathrm{~b}$ \\
\hline
\end{tabular}

${ }^{*}$ not detected at limit of detection $0.2 \mathrm{~g} / 100 \mathrm{~g} ;{ }^{* *}$ data for water content were transformed with $\operatorname{ArcSin}(\mathrm{x})$ function; $n$ : number of samples in a group of winter stores produced by bees in each feeding period (early and late) from each type of syrup; one-way analysis of variance (ANOVA) with Duncan's test were used. Different letters $(\mathrm{a}, \mathrm{b}, \mathrm{c})$ at mean values indicate statistically significant differences at $p \leq 0.05$.

Table 4. Physicochemical composition of winter stores produced by bees fed with homemade saccharose syrup.

\begin{tabular}{|c|c|c|c|c|c|c|c|c|c|c|}
\hline \multirow{3}{*}{ Physicochemical Parameter } & \multicolumn{5}{|c|}{ Feeding Period in 2012} & \multicolumn{5}{|c|}{ Feeding Period in 2013} \\
\hline & \multirow{2}{*}{$\begin{array}{l}\text { Fresh } \\
\text { Syrup }\end{array}$} & \multicolumn{2}{|c|}{ Early $(n=5)$} & \multicolumn{2}{|c|}{ Late $(n=5)$} & \multirow{2}{*}{$\begin{array}{l}\text { Fresh } \\
\text { Syrup }\end{array}$} & \multicolumn{2}{|c|}{ Early $(n=5)$} & \multicolumn{2}{|c|}{ Late $(n=5)$} \\
\hline & & From-to & Mean & From-to & Mean & & From-to & Mean & From-to & Mean \\
\hline Water $(\%)$ & 3.93 & $17.1-18.7$ & $18.1 b^{* * * *}$ & $17.1-19.6$ & $18.5 b^{* * * *}$ & 37.8 & $15.7-16.8$ & $16.2 \mathrm{a}^{* * * *}$ & $16.0-17.5$ & $17.0 \mathrm{a}^{* * * *}$ \\
\hline Electrical conductivity $(\mathrm{mS} / \mathrm{cm})$ & 0.07 & $0.15-0.28$ & $0.22 \mathrm{a}$ & $0.18-0.22$ & $0.20 \mathrm{a}$ & 0.06 & $0.18-0.31$ & $0.20 \mathrm{a}$ & $0.10-0.31$ & $0.20 \mathrm{a}$ \\
\hline Free acids $(\mathrm{mval} / \mathrm{kg})$ & 0.5 & $12.3-24.2$ & $18.5 \mathrm{c}$ & $17.8-21.5$ & $19.8 \mathrm{c}$ & 0.2 & $9.3-17.1$ & $12.0 \mathrm{~b}$ & $4.4-11.1$ & $7.6 \mathrm{a}$ \\
\hline $\mathrm{pH}$ & 7.67 & $3.90-4.08$ & $4.00 \mathrm{a}$ & $3.92-4.01$ & $3.96 \mathrm{a}$ & 7.75 & $3.82-4.06$ & $3.96 a$ & $4.19-4.45$ & $4.29 \mathrm{~b}$ \\
\hline Fructose $(\mathrm{g} / 100 \mathrm{~g})$ & n.d. * & $30.6-33.9$ & $32.5 b$ & $30.8-32.9$ & $32.1 \mathrm{~b}$ & n.d. * & $32.2-34.5$ & $33.5 b$ & $24.9-30.7$ & $29.1 \mathrm{a}$ \\
\hline Glucose (g/100 g) & n.d. * & $24.9-28.3$ & $26.7 \mathrm{~b}$ & $26.5-28.6$ & $27.7 \mathrm{~b}$ & n.d. ${ }^{*}$ & $25.5-27.6$ & $26.7 \mathrm{~b}$ & $20.6-25.1$ & $23.7 \mathrm{a}$ \\
\hline Sucrose (g/100 g) & 62.7 & $7.2-17.2$ & $10.1 \mathrm{ab}$ & $7.4-12.2$ & $9.4 \mathrm{ab}$ & 64.6 & $5.7-11.0$ & $8.3 \mathrm{a}$ & $10.3-22.4$ & $13.9 \mathrm{~b}$ \\
\hline Maltose (g/100 g) & n.d. * & $3.3-3.9$ & $3.6 \mathrm{a}$ & $2.5-2.8$ & $2.7 \mathrm{a}$ & n.d. ${ }^{*}$ & $3.8-4.3$ & $4.0 \mathrm{a}$ & $2.6-3.4$ & $3.2 \mathrm{a}$ \\
\hline Erlose $(\mathrm{g} / 100 \mathrm{~g})$ & n.d. * & $4.2-5.8$ & $4.9 \mathrm{a}$ & $5.3-6.8$ & $6.0 \mathrm{~b}$ & n.d. ${ }^{*}$ & $5.5-6.5$ & $6.1 b$ & $4.3-6.3$ & $5.4 \mathrm{ab}$ \\
\hline Maltotriose $(\mathrm{g} / 100 \mathrm{~g})$ & n.d. * & n.d. * & - & n.d. * & - & n.d. ${ }^{*}$ & n.d. * & - & n.d. * & - \\
\hline $\begin{array}{l}\text { Total of fructose and glucose } \\
\qquad(\mathrm{g} / 100 \mathrm{~g})\end{array}$ & n.d.. * & $55.5-62.2$ & $59.2 b$ & $57.3-61.5$ & $59.9 b$ & n.d. * & $58.0-61.9$ & $60.2 \mathrm{~b}$ & $45.5-55.4$ & $52.8 \mathrm{a}$ \\
\hline Fructose to glucose ratio $(\mathrm{F}: \mathrm{G})$ & - & $1.20-1.23$ & $1.22 \mathrm{~b}$ & $1.15-1.16$ & $1.16 \mathrm{a}$ & - & $1.24-1.27$ & $1.25 c$ & $2.19-1.25$ & $1.22 \mathrm{~b}$ \\
\hline $\begin{array}{l}\text { Total of determined sugars } \\
\qquad(\mathrm{g} / 100 \mathrm{~g})\end{array}$ & 62.7 & $77.0-83.9$ & $79.2 \mathrm{ab}$ & $78.5-80.2$ & $79.5 \mathrm{ab}$ & 64.6 & $79.2-82.0$ & $81.0 \mathrm{~b}$ & $76.6-79.0$ & $77.7 \mathrm{a}$ \\
\hline $\begin{array}{c}\text { Maltodextrins (DP4-DP7) } \\
\text { (g/100 g) }\end{array}$ & n.d. ${ }^{* *}$ & n.d. ** & - & n.d. ** & - & n.d. & n.d. ${ }^{* *}$ & - & n.d. ${ }^{* *}$ & - \\
\hline $\begin{array}{l}\text { 5-hydroxymethylfurfural (HMF) } \\
\qquad(\mathrm{mg} / \mathrm{kg})\end{array}$ & n.d. & $0.5-1.6$ & 1.1 & $0.4-6.3$ & 1.9 & n.d. & $0.3-3.8$ & 1.4 & n.d. ${ }^{* *}$ & - \\
\hline
\end{tabular}

${ }^{*}$ not detected at limit of detection $0.2 \mathrm{~g} / 100 \mathrm{~g}$; ${ }^{* *}$ not detected at limit of detection $0.05 \mathrm{~g} / 100 \mathrm{~g}$; ${ }^{* * *}$ not detected at limit of detection 0.2 $\mathrm{mg} / \mathrm{kg}$; ${ }^{* * * *}$ data for water content were transformed with $\operatorname{ArcSin}(\mathrm{x})$ function; $n$ : number of samples in a group of winter stores produced by bees in each feeding period (early and late) from each type of syrup; one-way analysis of variance (ANOVA) with Duncan's test were used. Different letters $(\mathrm{a}, \mathrm{b}, \mathrm{c})$ at mean values indicate statistically significant differences at $p \leq 0.05$.

Trends in the changes of physicochemical parameters of winter stores produced by bees from Apikel 20 and Apifood syrups were similar to the stores made from the Apifortune syrup. These results are presented in Table 2 (starch syrup II) and Table 3 (starch syrup III).

There were no statistically significant differences in the total of fructose and glucose content and maltose content in the winter stores produced from each kind of starch syrup between different feeding periods (early and late) in both years of the study. In the case of other tested parameters, significant differences between different feeding periods were found, but they were not confirmed for all of the analyzed starch syrups in both years of the study. 


\subsection{Physicochemical Composition of Winter Stores Produced by Bees from Saccharose Syrups}

The results of our study confirmed that the sugar composition of the winter stores processed by bees fed with homemade saccharose syrup assured the liquid consistency of the stores during storage in honeybee combs (Table 4). These stores were characterized by adequate water content (not higher than $18.5 \%$ ), a high content of monosaccharides expressed as a total of fructose and glucose content $(58.0 \mathrm{~g} / 100 \mathrm{~g}$, on average), an adequate quantitative composition of these monosaccharides expressed as the ratio of fructose to glucose content $(\mathrm{F}: \mathrm{G}=1.21$, on average), and a low content of other disaccharides (saccharose, maltose) and the trisaccharide erlose, in total not exceeding $20 \mathrm{~g} / 100 \mathrm{~g}$. The HMF content was below the detection limit $(0.2 \mathrm{mg} / \mathrm{kg}$ ) or on a very low level (no higher than $2.0 \mathrm{mg} / \mathrm{kg}$ ).

There were no statistically significant differences in the water content as well as in the electrical conductivity of the winter stores processed by bees fed with homemade saccharose syrup between the feeding periods in both years of the study. In the case of other tested parameters, significant differences between the feeding periods were found, but they were not confirmed in both years of the study.

Similarly, the winter stores made from inverted saccharose syrup (Apiinvert) had an adequate water content (no higher than $18.6 \%$ ), monosaccharides content (the total of fructose and glucose content) $(68.0 \mathrm{~g} / 100 \mathrm{~g}$, on average), a proper ratio of these two monosaccharides ( $\mathrm{F}: \mathrm{G}=1.22$, on average), and other disaccharides (sucrose and maltose) (Table 5). These winter stores had a relatively low content of HMF, from 4.7 (2013) to $12.1 \mathrm{mg} / \mathrm{kg}$ (2012), on average.

Table 5. Physicochemical composition of winter stores produced by bees fed with inverted saccharose syrup (Apiinvert).

\begin{tabular}{|c|c|c|c|c|c|c|c|c|c|c|}
\hline \multirow{3}{*}{ Physicochemical Parameter } & \multicolumn{5}{|c|}{ Feeding Period in 2012} & \multicolumn{5}{|c|}{ Feeding Period in 2013} \\
\hline & \multirow{2}{*}{$\begin{array}{l}\text { Fresh } \\
\text { Syrup }\end{array}$} & \multicolumn{2}{|c|}{ Early $(n=5)$} & \multicolumn{2}{|c|}{ Late $(n=5)$} & \multirow{2}{*}{$\begin{array}{l}\text { Fresh } \\
\text { Syrup }\end{array}$} & \multicolumn{2}{|c|}{ Early $(n=5)$} & \multicolumn{2}{|c|}{ Late $(n=5)$} \\
\hline & & From-to & Mean & From-to & Mean & & From-to & Mean & From-to & Mean \\
\hline Water (\%) & 26.5 & $17.4-20.3$ & $18.2 \mathrm{ab} * * *$ & $17.8-19.0$ & $18.6 b^{* * *}$ & 26.9 & $15.0-18.1$ & $16.3 a^{* * *}$ & $15.0-17.5$ & $16.6 a^{* * *}$ \\
\hline Electrical conductivity $(\mathrm{mS} / \mathrm{cm})$ & 0.05 & $0.11-0.22$ & $0.20 \mathrm{a}$ & $0.09-0.15$ & $0.11 \mathrm{a}$ & 0.01 & $0.09-0.19$ & $0.15 \mathrm{a}$ & $0.05-0.28$ & $0.12 \mathrm{a}$ \\
\hline Free acids (mval $/ \mathrm{kg})$ & 5.9 & $12.6-22.4$ & $16.1 \mathrm{c}$ & $11.5-15.4$ & $13.3 \mathrm{bc}$ & 0.9 & $6.3-12.3$ & $10.1 \mathrm{~b}$ & $3.6-9.8$ & $5.7 \mathrm{a}$ \\
\hline $\mathrm{pH}$ & 3.80 & $3.88-4.04$ & $3.96 \mathrm{ab}$ & $3.78-3.95$ & $3.89 \mathrm{a}$ & 4.52 & $3.81-4.42$ & 3.98ab & $3.95-4.43$ & $4.11 \mathrm{~b}$ \\
\hline Fructose $(\mathrm{g} / 100 \mathrm{~g})$ & 35.5 & $38.4-40.3$ & $39.5 b$ & $36.3-39.6$ & $38.2 b$ & 28.0 & $32.9-39.3$ & $37.4 \mathrm{~b}$ & $33.0-35.1$ & $34.4 \mathrm{a}$ \\
\hline Glucose (g/100 g) & 29.8 & $30.9-32.8$ & $32.0 \mathrm{c}$ & $31.4-34.6$ & $32.9 \mathrm{c}$ & 22.7 & $29.4-30.8$ & $30.1 b$ & $26.4-27.8$ & $27.3 \mathrm{a}$ \\
\hline Sucrose (g/100 g) & 4.3 & $1.8-2.9$ & $2.3 \mathrm{a}$ & $1.8-2.3$ & $2.1 \mathrm{a}$ & 20.2 & $5.8-9.4$ & $7.3 b$ & $10.5-14.9$ & $11.7 \mathrm{c}$ \\
\hline Maltose (g/100 g) & n.d. * & $0.5-1.1$ & $0.8 \mathrm{a}$ & $0.8-3.1$ & $1.8 \mathrm{~b}$ & n.d. * & $2.3-2.8$ & $2.6 \mathrm{~b}$ & $0.8-3.0$ & $1.7 \mathrm{ab}$ \\
\hline Erlose $(\mathrm{g} / 100 \mathrm{~g})$ & n.d. * & n.d. * & - & n.d. * & - & n.d. * & n.d. ${ }^{*}-0.5$ & - & n.d. *-2.3 & - \\
\hline Maltotriose (g/100 g) & n.d. * & n.d. * & - & n.d. * & - & n.d. * & n.d. * & - & n.d. * & - \\
\hline $\begin{array}{l}\text { Total of fructose and glucose } \\
\qquad(\mathrm{g} / 100 \mathrm{~g})\end{array}$ & 65.3 & $69.3-73.0$ & $71.6 \mathrm{c}$ & $68.4-72.8$ & $71.2 \mathrm{c}$ & 50.7 & $62.7-70.1$ & $67.4 b$ & $59.4-62.8$ & $61.7 \mathrm{a}$ \\
\hline Fructose to glucose ratio $(\mathrm{F}: \mathrm{G})$ & 1.19 & $1.22-1.26$ & $1.23 b$ & $1.05-1.23$ & $1.16 \mathrm{a}$ & 1.12 & $1.10-1.29$ & $1.24 \mathrm{~b}$ & $1.24-1.27$ & $1.26 \mathrm{~b}$ \\
\hline $\begin{array}{l}\text { Total of determined sugars } \\
\qquad(\mathrm{g} / 100 \mathrm{~g})\end{array}$ & 69.6 & $72.7-76.7$ & $75.0 \mathrm{a}$ & $74.4-76.4$ & $75.7 \mathrm{a}$ & 70.9 & $71.7-81.7$ & $78.2 b$ & $75.1-80.9$ & $77.0 \mathrm{a}$ \\
\hline $\begin{array}{c}\text { Maltodextrins (DP4-DP7) } \\
\text { (g/100 g) }\end{array}$ & n.d. ${ }^{* *}$ & n.d. ${ }^{* *}$ & - & n.d. ${ }^{* *}$ & - & $\underset{* *}{\text { n.d. }}$ & n.d. ${ }^{* *}$ & - & n.d. ${ }^{* *}$ & - \\
\hline $\begin{array}{l}\text { 5-hydroxymethylfurfural (HMF) } \\
\text { (mg/kg) }\end{array}$ & 47.4 & $8.8-14.0$ & $11.9 \mathrm{~b}$ & $10.1-17.2$ & $12.3 b$ & 25.7 & $1.9-6.9$ & $4.2 \mathrm{a}$ & $2.7-8.2$ & $5.1 \mathrm{a}$ \\
\hline
\end{tabular}

${ }^{*}$ not detected at limit of detection $0.5 \mathrm{~g} / 100 \mathrm{~g} ;{ }^{* *}$ not detected at limit of detection $0.05 \mathrm{~g} / 100 \mathrm{~g}$; ${ }^{* * *}$ data for water content were transformed with $\operatorname{ArcSin}(x)$ function; $n$ : number of samples in a group of winter stores produced by bees in each feeding period (early and late) from each type of syrup; one-way analysis of variance (ANOVA) with Duncan's test were used. Different letters $(a, b, c)$ at mean values indicate statistically significant differences at $p \leq 0.05$.

There were no statistically significant differences in the water content and electrical conductivity of the winter stores produced by the bees fed with inverted saccharose syrup between the comparable feeding periods in both years of the study. In the case of the other tested parameters, significant differences between feeding periods were found, but they were not confirmed in both years of the study.

\subsection{Comparison of Physicochemical Parameters of Winter Stores Produced by Bees Fed with Different Types of Syrups}

The water content did not exceed $17.2 \%$ in the winter stores produced by bees fed with starch syrups and $18.6 \%$, on average, in the winter stores produced by bees fed with 
saccharose syrups (inverted sucrose syrup, homemade sucrose syrup) in both years of the research (Table 6). Electrical conductivity did not exceed 0.17 and $0.22 \mathrm{mS} / \mathrm{cm}, \mathrm{pH} 4.29$ and 4.00, and free acidity 16.4 and $19.8 \mathrm{mval} / \mathrm{kg}$, on average, in the stores made from starch and sucrose syrups, respectively. HMF concentration in the winter stores was much lower in comparison to the initial content of this compound in fresh syrups and did not exceed $14.3 \mathrm{mg} / \mathrm{kg}$. HMF content in the stores produced from starch syrup III after the early and late feeding of bees in 2013 was statistically higher compared to stores made from two other starch syrups and inverted saccharose syrup. It needs to be highlighted that concentration of HMF in the fresh starch syrup III (Apifood) was also higher $(51.2 \mathrm{mg} / \mathrm{kg}$ ) in comparison to its concentration in other starch syrups ( 39.5 and $30.1 \mathrm{mg} / \mathrm{kg}$ ) as well as in saccharose inverted syrup $(25.7 \mathrm{mg} / \mathrm{kg})$.

Table 6. Comparison of physicochemical composition of winter stores $(n=5)$ produced by bees fed with different types of syrup in 2012 and 2013.

\begin{tabular}{|c|c|c|c|c|c|c|c|}
\hline \multirow[b]{2}{*}{ Season } & \multirow[b]{2}{*}{$\begin{array}{c}\text { Feeding } \\
\text { Period }\end{array}$} & \multirow[b]{2}{*}{ Type of Syrup } & \multicolumn{5}{|c|}{ Physicochemical Parameter (Mean Value) } \\
\hline & & & $\begin{array}{c}\text { Water * } \\
(\%)\end{array}$ & $\begin{array}{c}\text { Electrical } \\
\text { Conductivity } \\
(\mathrm{mS} / \mathrm{cm})\end{array}$ & $\mathrm{pH}$ & $\begin{array}{c}\text { Free } \\
\text { Acidity } \\
\text { (mval/kg) }\end{array}$ & $\begin{array}{c}\mathrm{HMF} * * * \\
(\mathrm{mg} / \mathrm{kg})\end{array}$ \\
\hline \multirow{10}{*}{2012} & \multirow{5}{*}{ Early } & Sucrose syrup & $18.1 \mathrm{c}$ & $0.22 \mathrm{c}$ & 4.00 & $18.5 b$ & $1.1 \mathrm{a}$ \\
\hline & & Inverted sucrose syrup & $18.2 \mathrm{c}$ & $0.20 \mathrm{~b}$ & 3.96 & $16.1 b$ & $11.9 \mathrm{~d}$ \\
\hline & & Starch syrup I & $15.8 \mathrm{a}$ & $0.10 \mathrm{a}$ & 3.94 & $11.7 \mathrm{a}$ & $8.7 \mathrm{C}$ \\
\hline & & Starch syrup II & $16.7 \mathrm{ab}$ & $0.09 a$ & 3.97 & $10.9 \mathrm{a}$ & $3.2 \mathrm{ab}$ \\
\hline & & Starch syrup III & $17.1 \mathrm{bc}$ & $0.08 \mathrm{a}$ & 3.98 & $9.8 \mathrm{a}$ & $3.6 b$ \\
\hline & \multirow{5}{*}{ Late } & Sucrose syrup & $18.5 b$ & $0.20 \mathrm{~b}$ & $3.96 \mathrm{ab}$ & $19.8 b$ & $1.9 \mathrm{a}$ \\
\hline & & Inverted sucrose syrup & $18.6 b$ & $0.11 \mathrm{a}$ & $3.89 a$ & $13.3 \mathrm{a}$ & $12.3 \mathrm{c}$ \\
\hline & & Starch syrup I & $17.2 \mathrm{a}$ & $0.13 a$ & $3.96 \mathrm{ab}$ & $14.3 \mathrm{a}$ & $11.7 \mathrm{c}$ \\
\hline & & Starch syrup II & $16.9 \mathrm{a}$ & $0.16 \mathrm{ab}$ & $3.93 \mathrm{ab}$ & $16.4 \mathrm{ab}$ & $4.3 \mathrm{ab}$ \\
\hline & & Starch syrup III & $16.2 \mathrm{a}$ & $0.12 \mathrm{a}$ & $3.98 \mathrm{~b}$ & $12.0 \mathrm{a}$ & $5.7 \mathrm{~b}$ \\
\hline \multirow{10}{*}{2013} & \multirow{5}{*}{ Early } & Sucrose syrup & $16.2 \mathrm{a}$ & $0.20 \mathrm{~b}$ & $3.96 \mathrm{ab}$ & $12.0 \mathrm{~b}$ & $1.4 \mathrm{a}$ \\
\hline & & Inverted sucrose syrup & $16.3 a$ & $0.15 \mathrm{ab}$ & $3.98 \mathrm{~b}$ & $10.1 \mathrm{ab}$ & $4.2 b$ \\
\hline & & Starch syrup I & $16.1 \mathrm{a}$ & $0.14 \mathrm{ab}$ & $3.90 \mathrm{ab}$ & $8.5 \mathrm{ab}$ & $5.6 b$ \\
\hline & & Starch syrup II & $15.6 \mathrm{a}$ & $0.09 a$ & $3.80 \mathrm{a}$ & $6.8 \mathrm{a}$ & $5.7 \mathrm{~b}$ \\
\hline & & Starch syrup III & $16.6 a$ & $0.15 \mathrm{ab}$ & $3.88 \mathrm{ab}$ & $9.9 \mathrm{ab}$ & $10.2 \mathrm{c}$ \\
\hline & \multirow{5}{*}{ Late } & Sucrose syrup & $17.0 \mathrm{~b}$ & $0.20 \mathrm{a}$ & $4.29 a$ & $7.6 a$ & n.d. $* *$ \\
\hline & & Inverted sucrose syrup & $16.6 \mathrm{ab}$ & $0.12 \mathrm{a}$ & $4.11 \mathrm{a}$ & $5.7 \mathrm{a}$ & $5.1 \mathrm{a}$ \\
\hline & & Starch syrup I & $15.6 \mathrm{a}$ & $0.13 \mathrm{aa}$ & $4.16 \mathrm{a}$ & $6.3 a$ & $7.9 \mathrm{a}$ \\
\hline & & Starch syrup II & $16.9 b$ & 0.17 & $4.20 \mathrm{a}$ & $7.8 \mathrm{a}$ & $8.3 a$ \\
\hline & & Starch syrup III & $16.5 \mathrm{ab}$ & $0.17 \mathrm{a}$ & $4.25 \mathrm{a}$ & $7.2 \mathrm{a}$ & $14.3 \mathrm{~b}$ \\
\hline
\end{tabular}

* data for water content were transformed with $\operatorname{ArcSin}(\mathrm{x})$ function; ** not detected at limit of detection $0.2 \mathrm{mg} / \mathrm{kg}$; $n$ : number of samples of winter stores produced by bees in each feeding period (early and late) from each type of syrup; one-way analysis of variance (ANOVA) with Duncan's test were used. Different letters $(\mathrm{a}, \mathrm{b}, \mathrm{c}, \mathrm{d})$ at mean values indicate statistically significant differences at $p \leq 0.05$; *** 5-hydroxymethylfurfural (HMF).

The total content of monosaccharides (the total of fructose and glucose) and the ratio of fructose to glucose content $(\mathrm{F}: \mathrm{G})$ of the winter stores produced by bees from starch syrups was statistically lower than in the winter stores produced by bees from sucrose syrups after the early and late feeding period in both years of the experiment (Table 7). Disaccharide sucrose was determined in saccharose syrups. In 2012, the content of sucrose in winter stores produced by bees fed with homemade saccharose syrup was statistically higher than that in the winter stores produced by bees fed with inverted sucrose syrup in both feeding periods, regardless of the feeding period. There were no statistically significant differences in the content of sucrose between the comparable feeding periods in 2013. 
Table 7. Comparison of sugars composition in winter stores $(n=5)$ produced by bees fed with different type of syrups in 2012 and 2013.

\begin{tabular}{|c|c|c|c|c|c|c|c|c|c|c|c|c|}
\hline \multirow[b]{2}{*}{ Season } & \multirow[b]{2}{*}{$\begin{array}{c}\text { Feeding } \\
\text { Period }\end{array}$} & \multirow[b]{2}{*}{ Type of Syrup } & \multicolumn{10}{|c|}{ Sugar (Mean Value) (g/100 g) } \\
\hline & & & Fructose & Glucose & Sucrose & Maltose & Erlose & $\begin{array}{l}\text { Malto- } \\
\text { triose }\end{array}$ & $\begin{array}{c}\text { Total of } \\
F+G\end{array}$ & $\begin{array}{c}\text { Glucose to } \\
\text { Fructose } \\
\text { Ratio (F:G) }\end{array}$ & $\begin{array}{c}\text { Total of } \\
\text { Determined } \\
\text { Sugars }\end{array}$ & $\begin{array}{l}\text { Maltodextrins } \\
\text { (DP4-DP7) }\end{array}$ \\
\hline \multirow{10}{*}{2012} & \multirow{5}{*}{ Early } & \multirow{5}{*}{$\begin{array}{l}\text { Sucrose syrup } \\
\text { Inverted sucrose } \\
\text { syrup } \\
\text { Starch syrup I } \\
\text { Starch syrup II } \\
\text { Starch syrup III }\end{array}$} & $32.5 \mathrm{c}$ & $26.7 a$ & $10.1 \mathrm{~b}$ & $3.6 \mathrm{~b}$ & 4.9 & n.d. * & $59.2 d$ & $1.22 \mathrm{c}$ & $79.2 \mathrm{e}$ & n.d. ** \\
\hline & & & $39.5 \mathrm{~d}$ & $32.0 \mathrm{~d}$ & $2.3 \mathrm{a}$ & $0.8 \mathrm{a}$ & n.d. * & n.d. * & $71.6 \mathrm{e}$ & $1.23 \mathrm{c}$ & $75.0 \mathrm{~d}$ & n.d. $* *$ \\
\hline & & & $21.5 b$ & $28.7 \mathrm{~b}$ & n.d. * & $12.7 \mathrm{c}$ & n.d. * & $2.2 \mathrm{a}$ & $48.2 b$ & $0.75 b$ & $65.6 \mathrm{a}$ & $4.9 \mathrm{c}$ \\
\hline & & & $13.7 \mathrm{a}$ & $26.4 a$ & n.d. * & $23.1 \mathrm{e}$ & n.d. * & $3.2 \mathrm{a}$ & $40.2 a$ & $0.52 a$ & $66.4 \mathrm{a}$ & $3.7 \mathrm{~b}$ \\
\hline & & & $21.3 b$ & $30.3 c$ & n.d. * & $14.8 \mathrm{~d}$ & n.d. $*$ & $2.3 \mathrm{a}$ & $51.6 \mathrm{c}$ & $0.70 \mathrm{~b}$ & 69.1c & $3.4 \mathrm{a}$ \\
\hline & \multirow{5}{*}{ Late } & \multirow{5}{*}{$\begin{array}{c}\text { Sucrose syrup } \\
\text { Inverted sucrose } \\
\text { syrup } \\
\text { Starch syrup I } \\
\text { Starch syrup II } \\
\text { Starch syrup III }\end{array}$} & $32.1 \mathrm{c}$ & $27.7 \mathrm{a}$ & $9.4 \mathrm{~b}$ & $2.7 \mathrm{a}$ & 6.0 & n.d. * & $59.9 b$ & $1.16 \mathrm{c}$ & $79.5 \mathrm{~d}$ & n.d. $* *$ \\
\hline & & & $38.2 \mathrm{~d}$ & $32.9 \mathrm{~b}$ & $2.1 \mathrm{a}$ & $1.8 \mathrm{a}$ & n.d. * & n.d. * & $71.2 \mathrm{c}$ & $1.16 \mathrm{c}$ & $75.7 \mathrm{c}$ & n.d. ${ }^{* *}$ \\
\hline & & & $21.6 b$ & $28.1 \mathrm{a}$ & n.d. ${ }^{*}$ & $13.3 b$ & n.d. * & $4.7 \mathrm{a}$ & $49.7 \mathrm{a}$ & $0.77 b$ & $67.6 \mathrm{a}$ & $4.5 b$ \\
\hline & & & $18.3 a$ & $27.0 \mathrm{a}$ & n.d. ${ }^{*}$ & $21.1 \mathrm{c}$ & n.d. * & $6.0 \mathrm{a}$ & $45.3 a$ & $0.67 a$ & $77.9 \mathrm{c}$ & $3.3 a$ \\
\hline & & & $20.9 \mathrm{ab}$ & $28.2 \mathrm{a}$ & n.d. ${ }^{*}$ & $18.5 \mathrm{c}$ & n.d. * & $5.1 \mathrm{a}$ & $49.2 \mathrm{a}$ & $0.74 a b$ & $73.1 \mathrm{~b}$ & $3.5 \mathrm{a}$ \\
\hline \multirow{10}{*}{2013} & \multirow{5}{*}{ Early } & \multirow{5}{*}{$\begin{array}{l}\text { Sucrose syrup } \\
\text { Inverted sucrose } \\
\text { syrup } \\
\text { Starch syrup I } \\
\text { Starch syrup II } \\
\text { Starch syrup III }\end{array}$} & $33.5 \mathrm{~d}$ & $26.7 a$ & $8.3 a$ & $4.0 \mathrm{a}$ & 6.1 & n.d. * & $60.2 d$ & $1.25 \mathrm{~d}$ & $81.0 \mathrm{~d}$ & n.d. $* *$ \\
\hline & & & $37.4 \mathrm{e}$ & $30.1 b c$ & $7.3 \mathrm{a}$ & $2.6 a$ & n.d. * & n.d. * & $67.4 \mathrm{e}$ & $1.24 \mathrm{~d}$ & $78.2 \mathrm{~d}$ & n.d. ** \\
\hline & & & $21.6 \mathrm{~b}$ & $29.5 b c$ & n.d. ${ }^{*}$ & $11.9 \mathrm{~b}$ & n.d. * & $5.0 \mathrm{a}$ & $51.2 \mathrm{~b}$ & $0.73 b$ & $67.4 \mathrm{a}$ & $4.8 \mathrm{c}$ \\
\hline & & & $16.0 \mathrm{a}$ & $29.1 \mathrm{~b}$ & n.d. * & $18.4 \mathrm{c}$ & n.d. * & $6.7 \mathrm{c}$ & $45.1 \mathrm{a}$ & $0.55 a$ & $70.5 \mathrm{~b}$ & $4.2 \mathrm{~b}$ \\
\hline & & & $24.2 \mathrm{c}$ & $30.9 c$ & n.d. ${ }^{*}$ & $12.0 \mathrm{~b}$ & n.d. * & $5.8 \mathrm{~b}$ & $55.1 \mathrm{c}$ & $0.78 \mathrm{c}$ & $72.9 \mathrm{c}$ & $2.9 a$ \\
\hline & \multirow{5}{*}{ Late } & \multirow{5}{*}{$\begin{array}{c}\text { Sucrose syrup } \\
\text { Inverted sucrose } \\
\text { syrup } \\
\text { Starch syrup I } \\
\text { Starch syrup II } \\
\text { Starch syrup III }\end{array}$} & $29.1 \mathrm{~d}$ & $23.7 \mathrm{a}$ & $13.9 \mathrm{a}$ & $3.2 \mathrm{a}$ & 5.4 & n.d. * & $52.8 \mathrm{bc}$ & $1.22 \mathrm{c}$ & $77.7 \mathrm{c}$ & n.d. $* *$ \\
\hline & & & $34.4 \mathrm{e}$ & $27.3 a$ & $11.7 \mathrm{a}$ & $1.7 \mathrm{a}$ & n.d. * & n.d. * & $61.7 \mathrm{~d}$ & $1.26 \mathrm{c}$ & $77.0 \mathrm{c}$ & n.d. ** \\
\hline & & & $22.4 \mathrm{~b}$ & $28.6 \mathrm{ab}$ & n.d. * & $11.6 \mathrm{~b}$ & n.d. * & $4.5 \mathrm{a}$ & $50.9 \mathrm{~b}$ & $0.78 \mathrm{ab}$ & $69.6 \mathrm{a}$ & $4.4 \mathrm{a}$ \\
\hline & & & $20.0 \mathrm{a}$ & $27.7 \mathrm{a}$ & n.d. * & $13.9 \mathrm{c}$ & n.d. * & $5.7 \mathrm{~b}$ & $47.7 \mathrm{a}$ & $0.72 \mathrm{a}$ & $72.3 \mathrm{~b}$ & $3.2 \mathrm{a}$ \\
\hline & & & $24.7 \mathrm{c}$ & $29.6 \mathrm{~b}$ & n.d. * & $11.5 \mathrm{~b}$ & n.d. * & $4.5 \mathrm{a}$ & $54.3 \mathrm{c}$ & $0.84 \mathrm{~b}$ & $74.4 \mathrm{~b}$ & $2.7 \mathrm{a}$ \\
\hline
\end{tabular}

${ }^{*}$ not detected at limit of detection $0.5 \mathrm{~g} / 100 \mathrm{~g} ;{ }^{* *}$ not detected at limit od detection $0.05 \mathrm{~g} / 100 \mathrm{~g}$; F: fructose, G: glucose; $n$ : number of samples of winter stores produced by bees in each feeding period (early and late) from each type of syrup; one-way analysis of variance (ANOVA) with Duncan's test were used. Different letters $(\mathrm{a}, \mathrm{b}, \mathrm{c})$ at mean values indicate statistically significant differences at $p \leq 0.05$.

Irrespective of the feeding period, in both years of the study, the winter stores produced by bees fed with starch syrups had statistically higher contents of maltose compared to the stores made from saccharose syrups. Trisaccharide maltotriose was determined in the winter stores produced by bees fed with starch syrups, while this sugar was not detected in the winter stores produced by bees fed with saccharose syrups. In 2013, depending on the feeding period as well as the type of starch syrup used, maltotriose content in the winter stores produced by bees fed with starch syrups was statistically different.

Maltodextrins were determined in starch syrups and winter stores produced by bees fed with these syrups; however, these components were not found (with a detection limit of $0.05 \mathrm{~g} / 100 \mathrm{~g}$ ) in the winter stores produced by bees from saccharose syrups. Moreover, quantitative results for maltodextrins in the winter stores produced by bees from starch syrups statistically differed between feeding periods in both years of the study.

\section{Discussion}

It is commonly known that different types of ready-to-use syrups (inverted saccharose syrups, starch syrups, and HFCSs) recommended for the winter feeding of bee colonies have to comprise a proper physicochemical composition. This is important for the assurance of the liquid consistency of winter stores, as this guarantees the proper overwintering and dynamic spring development of honeybee colonies $[5-9,16]$. There are several important physicochemical parameters of syrups that should be taken into consideration before feeding the bees: water content, sugars composition, HMF content, $\mathrm{pH}$, free acidity, and mineral contents. When fructose predominates over glucose in the monosaccharides composition, the winter stores do not crystallize in the cells of honeybee combs. From this point of view, the ratio of fructose to glucose content should be higher than 1.00 and the most beneficial value of this parameter is around 1.26 [16].

The results from our laboratory are compared with our previously published field results [12] and with the results received by other authors [8-10,17,23,24,30]. Our study included the physicochemical composition of syrups which were investigated also for 
their suitability for the winter feeding of honeybee colonies under field conditions [12]. The laboratory studies showed that the physicochemical properties of the winter stores is different from the composition of the syrups from which winter stores were made. Moreover, the composition of the winter stores depended on the composition of the fresh starch syrups, as well as on the weather condition during the feeding of the bee colonies.

Considering the shelf life of the winter stores, water content and $\mathrm{pH}$ seem to be very important. The average water content determined in our winter stores produced by the bees fed with starch and saccharose syrups did not exceed $18.6 \%$ and the average $\mathrm{pH}$ was around 4.00 , guaranteeing the stability of the winter stores in honeybee combs during overwintering and the spring development of the bee colonies.

Apart from the quantitative share of fructose and glucose, the crystallization of winter stores in the cells of honeybee combs depended on the malto-compounds (maltodextrins) content. Regarding crystallization, studies conducted by Rybak-Chmielewska [9] showed that the winter stores produced by bees from starch products in which crystallization occurred had a significantly lower content of fructose compared to glucose ( $\mathrm{F}: \mathrm{G}=0.50$, on average). In our study, a low fructose content compared to glucose in the winter stores produced by bees from starch syrups (F:G from 0.52 to 0.84 ) might suggest the crystallization of the winter stores in the honeybee combs during wintering. The high content of maltose and maltotriose in starch syrups can also be an additional factor contributing to the crystallization of the winter stores. These two sugars were partially hydrolyzed to glucose by bees' enzymes, resulting in an increase in glucose content. In our study, the glucose content in the winter stores averaged as much as $31 \mathrm{~g} / 100 \mathrm{~g}$. According to Ohe and Schönberger [3], the critical point at which a solution becomes saturated with glucose is $32 \mathrm{~g} / 100 \mathrm{~g}$.

The liquid consistency of starch products also depends on the malto-compounds content preventing the unwanted crystallization of glucose [31-33]. Enzyme $\alpha$-amylase hydrolyzes very long chains of amylose and amylopectin into glucose [34]. Amylose (a non-ramified form of starch) is made up of glucose subunits which are bound together with $\alpha-1,4$-glycoside bonds. Amylopectin is a ramified form of starch containing one $\alpha-1,6$-glycoside bond per ca. thirty $\alpha-1,4$-glycoside bonds. Intermediate products of this reaction are malto-compounds consisting of a few glucose molecules. In starch syrups, we identified maltodextrins containing from 4 to 7 glucose molecules (DP4-DP7) at a level of $4.0 \mathrm{~g} / 100 \mathrm{~g}$, on average. Based on the comparison of the data for starch syrups before and after processing by bees, it can be said that there was a slightly lower content of maltodextrin (DP4-DP7) in the winter stores produced by bees from these syrups $(2.7-4.9 \mathrm{~g} / 100 \mathrm{~g}$ ). Despite the unfavorable ratio of fructose to glucose content (below 0.80) and the high glucose content (especially in stores from starch syrup III in which the concentration of this sugar averaged between 30 and $31 \mathrm{~g} / 100 \mathrm{~g}$ in the early feeding period in both years of the study) it seems that the presence of maltodextrins prevented the crystallization of the winter stores in honeybee comb cells. The liquid consistency of the winter stores ensures that bees can use the food accumulated in the honeybee comb cells, having a beneficial effect on overwintering and the spring development of bee colonies.

Our field experiment confirmed that the problem of crystallization of winter stores produced by bees from starch syrups did not occur during and directly after overwintering [12]. Only in May 2013 was a low amount of crystallized syrups $(<0.2 \mathrm{~kg})$ as residues of winter food in bee colonies fed with starch syrups and in the control groups (fed with inverted saccharose syrup and homemade saccharose syrup) found.

However, it should be noted that some authors indicate that longer oligosaccharides in starch syrups may have a negative impact on overwintering of bees, especially in the case of a long and cold winter [7]. Worker bees can use soluble starch as an energy source for flight. The $\alpha$-amylase enzyme is also present in the gland secretions of older worker bees and those bees are capable of using it to generate energy [30]. Nonetheless, after analyzing the data of Rybak-Chmielewska et al. [8] it becomes evident that the individual malto-compounds underwent a significant reduction in the stores (by ca. 1/3). However, 
some of these compounds might overload bees' simple intestines during the long winter, possibly causing the colony losses during the difficult conditions of the winter of 2005/2006. The weather conditions during the winter of 2005/2006 differed significantly from the weather conditions of the two consecutive seasons (2012 and 2013) in which we performed this experiment [12]. The average temperature in winter of $2012 / 2013$ was $-0.7^{\circ} \mathrm{C}$, and in the winter season of $2012 / 2014+1.4^{\circ} \mathrm{C}$, while during the winter of $2005 / 2006$ the temperature dropped below $-20{ }^{\circ} \mathrm{C}$.

A very important issue concerns $\mathrm{HMF}$, which is considered to be harmful to bees $[10,17,23,24]$. Experiments on HMF toxicity to bees have been conducted under laboratory conditions. Although we have not studied the effect of HMF on bees directly, based on laboratory results we found that the concentration of HMF in some syrups used to feed the bees in our study was quite high. Therefore, there was a potential risk to the bees related to the toxicity of HMF. Some manufacturers declare wide range of HMF content $(20-40 \mathrm{mg} / \mathrm{kg}$ ) in products recommended for the winter feeding of bees (Apikel 20, Apifood), however, some of them do not specify values for this parameter (Apifortune, Apiinvert). An HMF level of $30 \mathrm{mg} / \mathrm{kg}$ can be considered as safe for bees [17]. The HMF content determined in the starch syrups used in our experiment ranged on average from 14.6 to $51.2 \mathrm{mg} / \mathrm{kg}$, and in the inverted saccharose syrup from 25.7 to $47.4 \mathrm{mg} / \mathrm{kg}$. Concentrations of this component in the winter stores was much lower, on average ranging from 3.2 to $14.3 \mathrm{mg} / \mathrm{kg}$ in the stores made from starch syrups and from 4.2 to $12.3 \mathrm{mg} / \mathrm{kg}$ in the stores made from inverted saccharose syrup. Although the initial HMF content in some of syrups was high, we did not notice any harmful effects on the bees.

Both the electrical conductivity value and the ash percentage are related to the mineral contents in the products used by bees as carbohydrate foods. In our studies, the electrical conductivity of the analyzed fresh syrups and winter stores produced by bees from those syrups was at a low level, not exceeding $0.2 \mathrm{mS} / \mathrm{cm}$. This value is comparable with values for monofloral honeys such as acacia, rape, citrus, Hedysarum, Lavandula, Phacelia, Rhododendron, and Rosmarinus honey [19,28,35]. According to some authors, mineral contents in the nectar honey used by bees as a carbohydrate food are at a safe level for bees $[2,16]$. Therefore, we can conclude that the winter stores for which the electrical conductivity does not exceed the value of $0.2 \mathrm{mS} / \mathrm{cm}$ are safe for bees.

Despite the low ratio of fructose to glucose content $(\mathrm{F}: \mathrm{G})$, the high content of HMF, and the low percentage content of maltodextrins (DP4-DP7) in the winter stores produced by bees from the tested starch syrups, the condition of the bee colonies (measured by strength: number of combs covered by bees and brood area after the end of supplementation), was similar to the condition of bee colonies fed with inverted sucrose syrup and homemade sucrose syrup [12]. Additionally, no statistically significant differences in the bee colony conditions were found when comparing feeding periods in the two consecutive seasons (2012 and 2013). The number of dead bees after overwintering in the bee colonies fed earlier (from the middle of August to the end of the first week of September) and later (from the first to second week of September to the end of the first week of October), as well as the consumption of syrups, did not differ statistically depending on the type of syrup used for feeding. The spring development of bee colonies assessed based on the increase in brood area between two measurements (taken at three-week intervals) was similar in all the compared groups and there was no significant differences between different foods and feeding periods.

Comparison of the data of the winter stores with natural honey allowed us to understand the differences between the honey obtained from natural sources [35-40] and from saccharose and starch syrups. Some authors showed that the products processed by bees fed with inverted (acid and heat treatment) saccharose syrups had a higher HMF content and a lower diastase activity, lower moisture content, and lower free acidity than natural honey and honey produced by the supplementary feeding of bees by saccharose syrup [36]. Saccharose content in two kinds of honey studied by these authors was higher than in natural honey. Our study showed that winter stores processed by bees from saccharose 
and inverted saccharose syrups contained a much higher content of saccharose compared to the results achieved by other authors for natural honey $[19,35-40]$. The winter stores processed by bees from starch syrups were characterized by a higher content of maltose and maltotriose and a lower content of simple sugars (fructose and glucose). Among the simple sugars, glucose was the dominant sugar, whereas in most samples of monofloral and multifloral honey fructose was the dominant sugar [19,35-40]. The winter stores processed by bees from starch syrups also contained starch maltodextrins, which are not natural ingredients of honey. It should be noted that the presence of maltodextrins in spring honey obtained from the colonies fed with starch syrups indicates that this honey may contain residues of the winter stores processed by bees from starch syrups [41]. Therefore, the collection of the first spring honey must be carried out following the art of beekeeping. Maltodextrins can be an indicator of honey adulteration with winter stores produced by bees fed by starch syrups, or even with the starch syrups themselves.

In conclusion, our studies confirmed that starch syrups can be recommended as winter food for bees. These are products of incomplete enzyme hydrolysis of starch containing, in addition to glucose and fructose, some amounts of maltose, maltotriose, and maltodextrins (DP4-DP7). The sugars composition of the tested starch syrups ensures the correct composition of the winter stores produced by the bees. The lower percentage content of starch maltodextrins (DP4-DP7) in these syrups guarantees the liquid consistency of the winter stores. A water content not exceeding $17.2 \%$ and a low $\mathrm{pH}$ value (about 4.0 ) ensure the stability of the stores in honeybee combs during overwintering and the spring development of bee colonies. We did not find significant differences in the physicochemical parameters of the winter stores depending on the feeding period over two consecutive seasons (2012 and 2013).

The results of our study show that the presence of malto-compounds (DP4-DP7) at a relatively low level (no higher than $5 \mathrm{~g} / 100 \mathrm{~g}$ ) and HMF content of up to $50 \mathrm{mg} / \mathrm{kg}$, in winter stores does not have negative impact on overwintering and the spring development of honeybee colonies.

Author Contributions: Conceptualization and methodology, T.S., E.W., P.S. (Piotr Semkiw), and P.S. (Piotr Skubida); investigation, P.S. (Piotr Semkiw), P.S. (Piotr Skubida), T.S., E.W., K.J., and M.W.; writing—original draft preparation, T.S. and E.W.; writing—review \& editing, T.S., E.W., P.S. (Piotr Semkiw), P.S. (Piotr Skubida), K.J., and M.W.; visualization, T.S., E.W., and P.S. (Piotr Semkiw); supervision, T.S.; All authors have read and agreed to the published version of the manuscript.

Funding: This research received no external funding.

Institutional Review Board Statement: Not applicable.

Informed Consent Statement: Not applicable.

Data Availability Statement: Not applicable.

Conflicts of Interest: The authors declare no conflict of interest.

\section{References}

1. Hrassnigg, N.; Crailsheim, K. Differences in drone and worker physiology in honeybees (Apis mellifera). Apidologie 2005, 36, 255-277. [CrossRef]

2. Brodschneider, R.; Crailsheim, K. Nutrition and health in honey bees. Apidologie 2010, 41, 278-294. [CrossRef]

3. Ohe von der, W.; Schönberger, H. Für die Ernährung der Bienen: Futtersirup im Vergleich. Dtsch. Bienen J. $2000,8,312-314$.

4. Ohe von der, W.; Schönberger, H. Bienenernährung: Futtersirup im Vergleich. Bienenvater 2002, 123, 11-15.

5. Rybak-Chmielewska, H.; Konopacka, Z. Co to jest izoglukoza? Pszczelarstwo 2005, 6, 6-7.

6. Ceksteryte, V.; Racys, J. The quality of syrups used for bee feeding before winter and their suitability for bee wintering. J. Apic. Sci. 2006, 50, 5-14. [CrossRef]

7. Rybak-Chmielewska, H.; Szczęsna, T.; Bieńkowska, M. Gas chromatograph (GC) study of sugar composition in honeys and winter stores processed by bees from sucrose syrups. J. Apic. Sci. 2006, 50, 147-155.

8. Rybak-Chmielewska, H.; Szczęsna, T.; Waś, E. Attempt to assay maltodextrins occurring in starch syrup and in winter stores made by bees from that syrup. J. Apic. Sci. 2006, 50, 127-135. 
9. Rybak-Chmielewska, H. High performance liquid chromatography (HPLC) study of sugar composition in some kinds of natural honey and winter stores processed by bees from starch syrup. J. Apic. Sci. 2007, 51, 23-38.

10. Leblanc, B.W.; Eggleston, G.; Sammataro, D.; Cornett, C.; Dufault, R.; Deeby, T.; Cyr, E.S. Formation of Hydroxymethylfurfural in Domestic High-Fructose Corn Syrup and Its Toxicity to the Honey Bee (Apis mellifera). J. Agric. Food Chem. 2009, 57, 7369-7376. [CrossRef] [PubMed]

11. Sammataro, D.; Weiss, M. Comparison of Productivity of Colonies of Honey Bees, Apis mellifera, Supplemented with Sucrose or High Fructose Corn Syrup. J. Insect Sci. 2013, 13, 1-13. [CrossRef]

12. Semkiw, P.; Skubida, P. Suitability of Starch Syrups for Winter Feeding of Honeybee Colonies. J. Apic. Sci. 2016, 60, 141-152. [CrossRef]

13. Matescu, C.; Savu, V.; Sapcaliu, A.; Radoi, I.; Negoita, C. The influence of corn syrup based solid food supplements during the inactive (winter) season upon the evaluation of major bacterial diseases in bees. Bul. Univ. Agric. Sci. Vet. Med. Anim. Sci. Biotechnol. 2015, 72, 169-172. [CrossRef]

14. Krainer, S.; Brodschneider, R.; Vollmann, J.; Crailsheim, K.; Riessberger-Gallé, U. Effect of hydroxymethylfurfural (HMF) on mortality of artificially reared honey bee larvae (Apis mellifera carnica). Ecotoxicology 2015, 25, 320-328. [CrossRef]

15. Abou-Shaara, H. Effects of various sugar feeding choices on survival and tolerance of honey bee workers to low temperatures. J. Ėntomol. Acarol. Res. 2017, 49, 6200. [CrossRef]

16. Konopacka, Z. Pokarmy do uzupełnienia zimowych zapasów rodzin pszczelich. Pszczelarstwo 2007, 7, 2-4.

17. Jachimowicz, T.; El Sherbiny, G. Zur Problematic der Verwendung von Invertzucker für die Bienenfütterung. Apidologie 1975, 6, 121-143. [CrossRef]

18. Capuano, E.; Fogliano, V. Acrylamide and 5-hydroksymethylfurfural (HMF): A review on metabolism, toxicity, occurrence in food and migration strategies. LWT 2011, 44, 793-810. [CrossRef]

19. De-Melo, A.A.M.; De Almeida-Muradian, L.B.; Sancho, M.T.; Pascual-Maté, A. Composition and properties of Apis mellifera honey: A review. J. Apic. Res. 2018, 57, 5-37. [CrossRef]

20. Fallico, B.; Arena, E.; Zappala, M. Degradation of 5-Hydroxymethylfurfural in Honey. J. Food Sci. 2008, 73, C625-C631. [CrossRef]

21. Fallico, B.; Arena, E.; Zappala, M. PREDICTION OF HONEY SHELF LIFE. J. Food Qual. 2009, 32, 352-368. [CrossRef]

22. Bailey, L. The Effect of Acid-Hydrolysed Sucrose on Honeybees. J. Apic. Res. 1966, 5, 127-136. [CrossRef]

23. Zirbes, L.; Nguyen, B.K.; De Graaf, D.C.; De Meulenaer, B.; Reybroeck, W.; Haubruge, E.; Saegerman, C. Hydroxymethylfurfural: A Possible Emergent Cause of Honey Bee Mortality? J. Agric. Food Chem. 2013, 61, 11865-11870. [CrossRef]

24. Gregorc, A.; Jurišić, S.; Sampson, B. Hydroxymethylfurfural Affects Caged Honey Bees (Apis mellifera carnica). Diversity 2019, 12, 18. [CrossRef]

25. Frizzera, D.; Del Fabbro, S.; Ortis, G.; Zanni, V.; Bortolomeazzi, R.; Nazzi, F.; Annoscia, D. Possible side effects of sugar supplementary nutrition on honey bee health. Apidologie 2020, 51, 594-608. [CrossRef]

26. Liebig, G. Getreidestärkesirup: Besser als sein Ruf. Dtsch. Bienen J. 2005, 13, 18-19.

27. Bogdanov, S.; Martin, P.; Lúllmann, C. Harmonized methods of the European Honey Commission. Apidologie 1997, 28, 1-59.

28. Szczęsna, T.; Rybak-Chmielewska, H.; Waś, E.; Kachaniuk, K.; Teper, D. Characteristic of Polish unifloral honeys. I. Rape honey (Brassica napus var. oleifera Metzger). J. Apic. Sci. 2011, 55, 111-119.

29. Statistica ver.10; StatSoft Inc.: Tulsa, OK, USA, 2011.

30. Hrassnigg, N.; Brodschneider, R.; Fleischmann, P.H.; Crailsheim, K. Unlike nectar foragers, honeybee drones (Apis mellifera) are not able to utilize starch as fuel for flight. Apidologie 2005, 36, 547-557. [CrossRef]

31. Chronakis, I.S. On the Molecular Characteristics, Compositional Properties, and Structural-Functional Mechanisms of Maltodextrins: A Review. Crit. Rev. Food Sci. Nutr. 1998, 38, 599-637. [CrossRef]

32. Ahmed, M.; Akter, M.S.; Chin, K.B.; Eun, J.B. Effect of maltodextrin concentration and drying temperature on quality properties of purple sweet potato flour. Food Sci. Biotechnolo. 2009, 18, 1487-1494.

33. Castro, N.; Durrieu, V.; Raynaud, C.; Rouilly, A. Influence of DE-value on the physicochemical properties of maltodextrin for melt extrusion processes. Carbohydr. Polym. 2016, 144, 464-473. [CrossRef]

34. Stryer, L. Węglowodany. In Biochemia; Wydawnictwo Naukowe PWN: Warszawa, Poland, 2003; pp. $494-514$.

35. Oddo, L.P.; Piro, R.; Bruneau, É.; Guyot-Declerck, C.; Ivanov, T.; Piskulová, J.; Flamini, C.; Lheritier, J.; Morlot, M.; Russmann, H.; et al. Main European unifloral honeys: Descriptive sheets. Apidologie 2004, 35, S38-S81. [CrossRef]

36. Özcan, M.; Arslan, D.; Ceylan, D.A. Effect of inverted saccharose on some properties of honey. Food Chem. 2006, 99, 24-29. [CrossRef]

37. Solayman, M.; Islam, A.; Paul, S.; Ali, Y.; Khalil, I.; Alam, N.; Gan, S.H. Physicochemical Properties, Minerals, Trace Elements, and Heavy Metals in Honey of Different Origins: A Comprehensive Review. Compr. Rev. Food Sci. Food Saf. 2016, 15, $219-233$. [CrossRef] [PubMed]

38. Di Marco, G.; Manfredini, A.; Leonardi, D.; Canuti, L.; Impei, S.; Gismondi, A.; Canini, A. Geographical, botanical and chemical profile of monofloral Italian honeys as food quality guarantee and territory brand. Plant. Biosyst. Int. J. Deal. All Asp. Plant. Biol. 2017, 151, 450-463. [CrossRef]

39. Ratiu, I.A.; Al-Suod, H.; Bukowska, M.; Ligor, M.; Buszewski, B. Correlation Study of Honey Regarding their Physicochemical Properties and Sugars and Cyclitols Content. Molecules 2019, 25, 34. [CrossRef] 
40. Truzzi, C.; Illuminati, S.; Annibaldi, A.; Finale, C.; Rossetti, M.; Scarponi, G. Physicochemical Properties of Honey from Marche, Central Italy: Classification of Unifloral and Multifloral Honeys by Multivariate Analysis. Nat. Prod. Commun. 2014, 9, 1595-1602. [CrossRef] [PubMed]

41. Szczęsna, T.; Skubida, P.; Semkiw, P. How winter stores produced by bees fed with starch syrups influence on honey quality? Unpublished work. 2021. 